\title{
CONSTRUCTION OF HAMILTON SEQUENCES FOR CERTAIN TEICHMÜLLER MAPPINGS
}

\author{
EDGAR REICH
}

(Communicated by Irwin Kra)

\begin{abstract}
Suppose $\sup \left\{\left|\iint_{|z|<1} \kappa(z) \varphi(z) d x d y\right|: \varphi(z)\right.$ analytic for $|z|<1$, $\left.\iint_{|z|<1}|\varphi(z)| d x d y=1\right\}=\operatorname{ess} \sup \{|\kappa(z)|:|z|<1\}$. The question of constructive determination of extremal sequences $\left\{\varphi_{n}\right\}$ is considered for some classes of functions $\kappa(z)$ that arise in connection with plane quasiconformal mappings. For example, such a sequence $\left\{\varphi_{n}\right\}$ is constructed explicitly for the $\kappa(z)$ that arises in connection with the affine stretch of Strebel's chimney domain.
\end{abstract}

1. Introduction. $f$ is a Teichmüller mapping of the unit disk, $U=\{|z|<1\}$, if $f$ is a quasiconformal mapping of $U$ with complex dilatation

$$
\frac{f_{\bar{z}}}{f_{z}}=k \frac{\overline{\phi(z)}}{|\phi(z)|}, \quad z \in U
$$

where $\phi(z)$ is holomorphic in $U$, and $k$ is a positive constant. Let $\mathfrak{B}(\Omega)$ denote the class of functions $\phi(z)$ holomorphic in a region $\Omega$, with the additional restriction that

$$
0<\|\phi(z)\|=\iint_{\Omega}|\phi(z)| d x d y<\infty, \quad z=x+i y .
$$

A necessary and sufficient condition that $f$ is an extremal mapping (among the class of quasiconformal mappings of $U$ with the same boundary values as $f$ ) is that [2] there exists a so-called Hamilton sequence, namely, a sequence $\phi_{n} \in \mathfrak{B}(U)$, such that

$$
\lim _{n \rightarrow \infty} \frac{\left|\iint_{U}(\overline{\phi(z)} /|\phi(z)|) \phi_{n}(z) d x d y\right|}{\left\|\phi_{n}(z)\right\|}=1 .
$$

If $\phi \in \mathfrak{B}(U)$, then, of course, $\phi_{n}(z)=\phi(z), n=1,2,3, \ldots$, constitutes a Hamilton sequence; so the problem of whether a Hamilton sequence exists is nontrivial only when $\|\phi(z)\|=\infty$. In principle, if a Hamilton sequence, $\left\{\phi_{n}\right\}$, does exist, such a sequence can be realized in terms of a sequence of "polygonal" Teichmüller mappings $f_{n}$ which agree with $f$ at finitely many boundary points (see $[2$, Theorem 6]). However, these $f_{n}$ 's are obtained by a highly nonconstructive process, with the result that the relationship of the properties of the corresponding sequence $\left\{\phi_{n}\right\}$ to the properties of $\phi$ is quite obscure. The question arises whether a Hamilton sequence, if one does exist, can be obtained in a more direct manner. In particular, we shall concern ourselves with the following possibility: If $\left\{R_{n}\right\}$ is a sequence of numbers,

Received by the editors April 23, 1987.

1980 Mathematics Subject Classification (1985 Revision). Primary 30C60, 30C75; Secondary $30 \mathrm{H} 05$. 
$0<R_{n}<1, \lim R_{n}=1$, does $\left\{\phi\left(R_{n} z\right)\right\}$ constitute a Hamilton sequence? We shall answer this question principally in the special case when $\phi(z)$ is holomorphic in $\bar{U}$ except for a finite number of poles on $\partial U$. In $\S 2$, we shall verify the following:

THEOREM 1. Suppose $\phi(z)$ is holomorphic for $|z| \leq 1$ except for a finite number of poles on $\{|z|=1\}$. Then

$$
\lim _{R \rightarrow 1} \frac{\left|\iint_{U}(\overline{\phi(z)} /|\phi(z)|) \phi(R z) d x d y\right|}{\|\phi(R z)\|}=1
$$

if and only if $\phi(z)$ has poles of at most order 2 on $\{|z|=1\}$.

As was first noted by Strebel [5], with his famous chimney region, an extremal Teichmüller mapping is not necessarily uniquely extremal. For the class of $\phi$ 's under consideration here, Sethares [4] showed that unique extremality obtains if and only if the poles are of the first or second order. Thus, Theorem 1 is equivalent to the following.

THEOREM $1^{\prime}$. Suppose $\phi(z)$ is holomorphic for $|z| \leq 1$ except for a finite number of poles on $\{|z|=1\}$. Then $\left\{\phi\left(R_{n} z\right)\right\}$ is a Hamilton sequence if and only if the Teichmüller mapping (1.1) is uniquely extremal.

The "if" part, above, is in fact known to hold for a somewhat larger class of $\phi$ 's. Examination of the proof of Theorem 2 of Hayman and Reich [1] shows that what is proved there is actually tantamount to the following.

THEOREM 2. Suppose $\phi(z)$ is holomorphic for $|z|<1$, and

$$
\int_{0}^{2 \pi}\left|\phi\left(r e^{i \theta}\right)\right| d \theta \leq \frac{1}{1-r}, \quad 0 \leq r<1 .
$$

Then the Teichmüller mapping (1.1) is uniquely extremal and $\left\{\phi\left(R_{n} z\right)\right\}$ is a Hamilton sequence.

The proof of Theorem 2 is complicated. Thus, even though Theorem 2 implies the "if" part of Theorem 1, we include a proof of the latter in $\S 2$ since only a short computation is involved.

In $\S 3$ we interpret our results for affine mappings of certain simply connected regions, and in $\S 4$ we consider the question of Hamilton sequences for the affine stretch of Strebel's chimney region.

2. Proof of Theorem 1. (a) Suppose $\|\phi(z)\|<\infty$ (as is the case if $\phi$ has at worst first order poles).

Given $\varepsilon>0$, choose $R_{0}$ such that

$$
\iint_{R_{0}^{2}<|z|<1}|\phi(z)| d x d y<\varepsilon .
$$

Then,

$$
\iint_{R_{0}<|z|<1}|\phi(R z)| d x d y=\frac{1}{R^{2}} \iint_{R_{0}} \iint_{R<|z|<R}|\phi(z)| d x d y<\frac{\varepsilon}{R_{0}^{2}}, \quad \text { if } R \geq R_{0} .
$$


Therefore,

$$
\left|\iint_{U} \frac{\overline{\phi(z)}}{|\phi(z)|} \phi(R z) d x d y\right| \geq\left|\iint_{|z|<R_{0}} \frac{\overline{\phi(z)}}{\phi(z)} \phi(R z) d x d y\right|-\frac{\varepsilon}{R_{0}^{2}}, \quad R \geq R_{0} .
$$

Since $\phi(R z)$ is bounded in $\left\{|z| \leq R_{0}\right\}$, uniformly with respect to $R, R_{0} \leq R<1$, we have

$$
\varliminf_{R \rightarrow 1}\left|\iint_{U} \frac{\overline{\phi(z)}}{|\phi(z)|} \phi(R z) d x d y\right| \geq \iint_{|z|<R_{0}}|\phi(z)| d x d y-\frac{\varepsilon}{R_{0}^{2}} .
$$

Hence

$$
\lim _{R \rightarrow 1}\left|\iint_{U} \frac{\overline{\phi(z)}}{|\phi(z)|} \phi(R z) d x d y\right|=\|\phi(z)\|
$$

Also,

$$
\lim _{R \rightarrow 1} \iint_{U}|\phi(R z)| d x d y=\|\phi(z)\|
$$

(b) Suppose $\phi(z)$ has second-order poles, but none of higher order.

$$
\begin{aligned}
& \iint_{U} \frac{\overline{\phi(z)}}{|\phi(z)|} \phi(R z) d x d y \\
&\|\phi(R z)\| R^{2}+R^{2} \frac{\iint_{|z|<R} \frac{\overline{\phi(z)}}{|\phi(z)|}[\phi(R z)-\phi(z)] d x d y}{\iint_{|z|<R}|\phi(z)| d x d y} \\
&+R^{2} \frac{\iint_{R<|z|<1} \frac{\overline{\phi(z)}}{|\phi(z)|} \phi(R z) d x d y}{\iint_{|z|<R}|\phi(z)| d x d y}
\end{aligned}
$$

The denominator on the right side goes to infinity as $R \rightarrow 1$. For the absolute values of the terms in the numerator, we have

$$
\begin{aligned}
I_{1}(R) & =\left|\iint_{|z|<R} \frac{\overline{\phi(z)}}{|\phi(z)|}[\phi(R z)-\phi(z)] d x d y\right| \leq \iint_{|z|<R}|\phi(R z)-\phi(z)| d x d y \\
& \leq \int_{0}^{R} r d r \int_{0}^{2 \pi} d \theta \int_{R r}^{r} d t\left|\phi^{\prime}\left(r e^{i \theta}\right)\right|, \\
I_{2}(R) & =\left|\iint_{R<|z|<1} \frac{\overline{\phi(z)}}{|\phi(z)|} \phi(R z) d x d y\right| \leq \frac{1}{R^{2}} \iint_{R^{2}<|z|<R}|\phi(z)| d x d y .
\end{aligned}
$$

Now, if $a>0, m>2$, then

$$
\int_{-a}^{a} \frac{d \theta}{\left|1-r e^{i \theta}\right|^{m}}=\frac{p}{(1-r)^{m-1}}+o\left(\frac{1}{(1-r)^{m-1}}\right), \quad \text { as } r \rightarrow 1(0<r<1) \text {, }
$$

where $p$ is a positive constant, not depending on $a$. Using (2.1), one finds that

$$
\lim _{R \rightarrow 1} I_{1}(R)=\lim _{R \rightarrow 1} I_{2}(R)=0
$$

and (1.3) therefore follows.

(c) Suppose the highest order poles of $\phi(z)$ are of order $m, m \geq 3$. We first derive a lemma. 
LEMMA. ${ }^{1}$ Suppose $H=\{\operatorname{Re} z>0\}, g \in \mathfrak{B}(H), \alpha>0$. Then

$$
\mid \iint_{H} e^{2 i(1-\alpha / \pi) \theta} g\left(r e^{i \theta)} d r d \theta\left|\leq \frac{|\sin \alpha|}{\alpha} \iint_{H}\right| g(z) \mid d x d y .\right.
$$

To prove this, let $w=(\alpha / \pi) \log z$ map $H$ onto the strip $\Sigma=\{w=u+i v:-\infty<$ $u<\infty,-\alpha / 2<v<\alpha / 2\}$. In $\Sigma$, define $h(w)$ by

$$
h(w) d w^{2}=g(z) d z^{2}
$$

that is,

$$
h(w)=\left(\pi^{2} / \alpha^{2}\right) e^{2 \pi w / \alpha} g\left(e^{\pi w / \alpha}\right)
$$

Then,

$$
A=\iint_{H} \frac{\overline{z^{\alpha / \pi}} z}{z^{\alpha / \pi} \bar{z}} g(z) d x d y=\int_{-\alpha / 2}^{\alpha / 2} e^{-2 i v} d v \int_{-\infty}^{\infty} h(u+i v) d u
$$

while

$$
B=\iint_{H}|g(z)| d x d y=\iint_{\Sigma}|h(w)| d u d v .
$$

Since $B<\infty$, it follows firstly, that $\int_{-\infty}^{\infty} h(u+i v) d u$ exists for a.a.v., $-\alpha / 2<v<$ $\alpha / 2$, and secondly, that there exist sequences $\left\{u_{n}\right\}, u_{n} \uparrow \infty$, and $\left\{u_{n}^{\prime}\right\}, u_{n}^{\prime} \downarrow-\infty$, such that

$$
\lim \int_{-\alpha / 2}^{\alpha / 2}\left|h\left(u_{n}+i v\right)\right| d v=\lim \int_{-\alpha / 2}^{\alpha / 2}\left|h\left(u_{n}^{\prime}+i v\right)\right| d v=0 .
$$

Since $h$ is analytic in $\Sigma$, we therefore conclude by Cauchy's theorem, that

$$
\int_{-\infty}^{\infty} h(u+i v) d u=c=\text { const for a.a.v. }
$$

Hence,

$$
A=c \int_{-\alpha / 2}^{\alpha / 2} e^{-2 i v} d v=-c \sin \alpha
$$

Also,

$$
|c| \leq \int_{-\infty}^{\infty}|h(u+i v)| d u \text { a.a.v. }
$$

Therefore $\alpha|c| \leq B$, and $|A| \leq(B / \alpha)|\sin \alpha|$, which proves (2.2).

Let $\alpha=((\mu-2) / 2) \pi$. If we $\operatorname{map}\{|\zeta|<1\}$ onto $\{\operatorname{Re} z>0\}$ and transfer $g(z) d z^{2}$ as a quadratic differential, (2.2) becomes

$$
\left|\iint_{U} \frac{|1+z|^{4-\mu}|1-z|^{\mu}}{(\overline{1+z})^{4-\mu}(\overline{1-z})^{\mu}} g(z) d x d y\right| \leq \frac{\mid \sin ((\mu-2) / 2) \pi) \mid}{((\mu-2) / 2) \pi} \iint_{U}|g(z)| d x d y,
$$

which holds whenever $\mu>2$, and $g \in \mathfrak{B}(U)$.

\footnotetext{
${ }^{1}$ This is a generalization of a result found in [3]. The bound is sharp for every $\alpha$.
} 
Turning now to the proof for case (c), by contradiction, suppose (1.3) held. By considering (2.1) we see that the effect of poles of order $m$ predominates as $R \rightarrow 1$. Say, there is a pole of order $m$ at $z=1$. Then it is necessarily true that

$$
\lim _{R \rightarrow 1} \frac{\left|\iint_{S} \frac{\overline{\phi(z)}}{|\phi(z)|} \phi(R z) d x d y\right|}{\iint_{S}|\phi(R z)| d x d y}=1
$$

for any set $S=\{|\arg z|<\delta, \rho<|z|<1\}(\delta>0,0<\rho<1)$.

The function $\phi(z)$ has the form

$$
\phi(z)=(z-1)^{-m} F(z)
$$

where $F(z)$ is holomorphic and, say, nonvanishing in $\bar{S}$. If $G(z)$ is any other function holomorphic and nonvanishing in $\bar{S}$, and $G(1)=F(1)$, and if $\phi$ is replaced by

$$
\psi(z)=(z-1)^{-m} G(z), \quad z \in S,
$$

then the quantities

$$
\iint_{S} \frac{\overline{\phi(z)}}{|\phi(z)|} \phi(R z) d x d y-\iint_{S} \frac{\overline{\psi(z)}}{|\psi(z)|} \psi(R x) d x d y
$$

and

$$
\iint_{S}|\phi(R z)-\psi(R z)| d x d y
$$

are negligible compared to $\iint_{S}|\phi(R z)| d x d y$ as $R \rightarrow 1$. Therefore (2.4) must hold with $\phi$ replaced by $\psi$. Moreover, if $\psi(z)$ is actually holomorphic in $\bar{U}$ except for poles on $\partial U$, and if all poles of $\psi$ on $\partial U$, except that at $z=1$, are of strictly lower order than $m$, then by the same reasoning as the one leading from (1.3) to (2.4), we conclude that, necessarily,

$$
\lim _{R \rightarrow 1} \mid \frac{\left|\iint_{U} \frac{\overline{\psi(z)}}{|\psi(z)|} \psi(R z) d x d y\right|}{\iint_{U}|\psi(R z)| d x d y}=1
$$

Choosing

$$
\psi(z)=\frac{(-1)^{m} 2^{4-m}}{(1+z)^{4-m}(1-z)^{m}}
$$

this contradicts (2.3), however.

3. Affine mappings. Let $\Omega$ be a simply connected planar region of hyperbolic type, but not necessarily of finite area $|\Omega|$. Introducing the auxiliary conformal mapping $z=F(\varsigma)$, of $\{|\varsigma|<1\}$ onto $\Omega$, and using (1.2), one notes that a necessary and sufficient condition that the affine stretch

$$
A_{k}(z)=K x+i y, \quad z \in \Omega, K>1, z=x+i y
$$

is an extremal mapping (among the class of quasiconformal mappings of $\Omega$ with the same prime-end boundary values as $A_{k}$ ) is that there exists a sequence $\phi_{n} \in \mathfrak{B}(\Omega)$, such'that

$$
\lim _{n \rightarrow \infty} \frac{\iint_{\Omega} \phi_{n}(z) d x d y}{\left\|\phi_{n}(z)\right\|}=1
$$


We refer to $\left\{\phi_{n}\right\}$ satisfying (3.1) as a Hamilton sequence for $\Omega$. If $|\Omega|<\infty$, then $\phi_{n}(z) \equiv 1, n=1,2, \ldots$, constitutes a Hamilton sequence, but when $|\Omega|=\infty$, the constant 1 does not belong to $\mathfrak{B}(\Omega)$, and, in fact, there may be no Hamilton sequence since $A_{k}$ need not be extremal. (This occurs, for example, when $\Omega$ is a half-plane.)

In order to form a putative Hamilton sequence for $\Omega$, take $0<R<1, R=R_{n} \uparrow 1$, and let

$$
\phi_{R}(z)=\Phi_{R}^{\prime}(z)^{2} \quad \text { where } \Phi_{R}(z)=F\left(R F^{-1}(z)\right), z \in \Omega \text {. }
$$

Since $\Phi_{R}$ maps $\Omega$ conformally onto a subregion of finite area, it is evident that $\phi_{R}(z) \in \mathfrak{B}(\Omega), 0<R<1$. Moreover, clearly,

$$
\lim _{R \rightarrow 1} \phi_{R}(z)=1 \quad \text { pointwise for all } z \in \Omega \text {. }
$$

The obvious question is: For which $\Omega$ is the putative sequence $\left\{\phi_{R}(z)\right\}$ actually a Hamilton sequence? We can say the following:

THEOREM 3. Suppose the mapping function $z=F(\varsigma)$ of $\{|\varsigma|<1\}$ onto $\Omega$ is such that $F^{\prime}(\varsigma)^{2}$ is holomorphic for $|\varsigma| \leq 1$ except for a finite number of poles on $\{|\varsigma|=1\}$. Then the putative sequence $\left\{\phi_{R}(z)\right\}, R \uparrow 1$, is a Hamilton sequence if and only if the poles are at most of order 2 , and this occurs if and only if $A_{K}(z)$ is a uniquely extremal quasiconformal mapping of $\Omega$.

ProOF. If we transfer $A_{K}(z)$ to $\{|\zeta|<1\}$ by means of $F$, the question becomes that of determining whether a Teichmüller mapping with complex dilatation

$$
k \frac{\overline{F^{\prime}(\varsigma)^{2}}}{\left|F^{\prime}(\varsigma)\right|^{2}} \quad\left(k=\frac{K-1}{K+1}\right)
$$

is extremal or uniquely extremal, as the case may be, and whether $\left\{R^{2} F^{\prime}\left(R_{\zeta}\right)^{2}\right\}$, $R=R_{n} \uparrow 1$, constitutes a corresponding Hamilton sequence. Since the factor $R^{2}$ is immaterial, the assertion is immediate by Theorem $1^{\prime}$.

EXAMPLE. Let $\Omega$ be the strip

$$
\Sigma_{0}=\{z=x+i y:-1<y<1\} .
$$

Here,

$$
F(\varsigma)=\frac{2}{\pi} \log \frac{1+\varsigma}{1-\varsigma}, \quad F^{\prime}(\varsigma)^{2}=\frac{16}{\pi^{2}(1+\varsigma)^{2}(1-\varsigma)^{2}} \quad(|\varsigma|<1) .
$$

By Theorem 3, $\left\{\phi_{R}(z)\right\}$, as defined by (3.2), is a Hamilton sequence. The fact that the affine stretch of $\Sigma_{0}$ is a uniquely extremal mapping was first proved in [5]. For application below, we note that a Hamilton sequence $\left\{\sigma_{n}(z)\right\}$ for $\Sigma_{0}$ can also be obtained explicitly as follows. Let

$$
\sigma_{n}(z)=(1 / n) \exp \left(-z^{2} / n^{2}\right) .
$$

One finds that

$$
\iint_{\Sigma_{0}} \sigma_{n}(z) d x d y=2 \sqrt{\pi}
$$


while

$$
\iint_{\Sigma_{0}}\left|\sigma_{n}(z)\right| d x d y=\sqrt{\pi} \int_{-1}^{1} \exp \left(\frac{y^{2}}{n^{2}}\right) d y \rightarrow 2 \sqrt{\pi} .
$$

It is evident that together with a Hamilton sequence $\left\{\phi_{n}(z)\right\}$ for $\Sigma_{0},\left\{\phi_{n}\left(z-\lambda_{n}\right)\right\}$ is also a Hamilton sequence, for any sequence of real numbers $\left\{\lambda_{n}\right\}$.

4. The chimney region. The chimney region

$$
\mathfrak{C}=\{z: \operatorname{Re} z<0\} \cup \Sigma_{0}
$$

was introduced by Strebel who showed, with extremal-length methods [5], that $A_{K}(z)$ is extremal for $\mathfrak{C}$ but not uniquely extremal.

In order to approach the problem of constructing Hamilton sequences for $\mathfrak{C}$ we need to first consider some qualitative properties of the functions mapping $\mathfrak{C}$ conformally onto a half-plane and a disk. In fact, these mappings can be expressed in "finite form" as Schwarz-Christoffel transformations in terms of elementary functions.

Let $z(\gamma)$ be a conformal mapping of the upper half-plane $\{\operatorname{Im} \gamma>0\}$ onto $\mathfrak{C}$. In view of the symmetry of $\mathfrak{C}$ we can preassign $z(-1)=-i, z(1)=i, z(0)=$ the point $\infty$ at the right end of $\Sigma_{0}, z(\infty)=$ the point $\infty$ in the half-plane $\{\operatorname{Re} z<0\}$. On the basis of the angles involved we find that

$$
\left(\frac{d z}{d \gamma}\right)^{2}=c \frac{1-\gamma^{2}}{\gamma^{2}}
$$

where $c$ is a positive constant. Since for every semicircle $0 \leq \arg \gamma \leq \pi,|\gamma|=h$, $(0<h<1)$, one has $\operatorname{Im} z(h)=1, \operatorname{Im} z(-h)=-1$, it follows that $c=4 / \pi^{2}$. Integrating, we obtain

$$
z=\frac{1}{\pi} \log \frac{\sqrt{1-\gamma^{2}}+1}{\sqrt{1-\gamma^{2}}-1}-\frac{2}{\pi} \sqrt{1-\gamma^{2}}, \quad \operatorname{Im} \gamma>0,
$$

where $\sqrt{1-\gamma^{2}}$ is the branch with positive real part, and the logarithm term goes to zero for $\gamma=i M, M \rightarrow+\infty$.

THEOREM 4. (i) Let $T(z)$ map $\mathfrak{C}$ conformally onto $\Sigma_{0}, T(i)=i, T(-i)=-i$, $T(+\infty)=+\infty$, and let $\left\{\sigma_{n}(z)\right\}$ be the Hamiltcn sequence (3.3) for $\Sigma_{0}$. Then

$$
q_{n}(z)=\sigma_{n}\left[T(z)-n^{3}\right]
$$

is a Hamilton sequence for $\mathfrak{C}$.

(ii) Let $F(\varsigma)$ map $\{|\varsigma|<1\}$ conformally onto $\mathfrak{C}$. The sequence (3.2), with $R=$ $R_{n} \uparrow 1, z \in \mathfrak{C}$, is not a Hamilton sequence for $\mathfrak{C}$.

ProOF (i). The mapping $T$ is given by

$$
w=T(z)=\frac{2}{\pi} \log \frac{i}{\gamma(z)} .
$$

Therefore,

$$
\left(\frac{d z}{d w}\right)^{2}=1+e^{-\pi w}, \quad w \in \Sigma_{0}
$$


Hence,

$$
\iint_{\mathfrak{C}} q_{n}(z) d x d y=\iint_{\Sigma_{0}} \sigma_{n}\left(w-n^{3}\right)\left|1+e^{-\pi w}\right| d u d v, \quad w=u+i v .
$$

A straightforward estimate shows that the choice of the term $n^{3}$ guarantees, together with the rapid decay of the $\sigma_{n}$ functions, that

$$
\lim _{n \rightarrow \infty} \iint_{\substack{w \in \Sigma_{0} \\ \operatorname{Re} w<A}} \sigma_{n}\left(w-n^{3}\right)\left|1+e^{-\pi w}\right| d u d v=0
$$

and that consequently the term $\left|1+e^{-\pi w}\right|$ behaves like the constant 1 in its effect as $n \rightarrow \infty$. Therefore, just as for (3.4), (3.5), one obtains

$$
\lim _{n \rightarrow \infty} \iint_{\mathfrak{C}} q_{n}(z) d x d y=\lim _{n \rightarrow \infty} \iint_{\mathfrak{C}}\left|q_{n}(z)\right| d x d y=2 \sqrt{\pi} .
$$

(ii) The mapping $F(\varsigma)$ is obtained by composition of $\gamma(z)$ with the Möbius transformation,

$$
i \gamma=(s-1) /(s+1)
$$

Therefore,

$$
F^{\prime}(\varsigma)^{2}=\frac{32}{\pi^{2}} \frac{\varsigma^{2}+1}{(\varsigma-1)^{2}(\varsigma+1)^{4}}
$$

Since there is a pole of fourth order, the putative sequence is not a Hamilton sequence in this case. Attention should be called to the fact that an alternative construction of a Hamilton sequence for the affine stretch of the chimney region is possible by analyzing the proof of Ortel's Theorem 1 in [6].

\section{REFERENCES}

1. W. K. Hayman and Edgar Reich, On Teichmüller mappings of the disk, Complex Variables 1 (1982), 1-12.

2. Edgar Reich and Kurt Strebel, Extremal quasiconformal mappings with given boundar values, Contributions to Analysis, a Collections of Papers Dedicated to Lipman Bers, Academic Press, New York, 1974, pp. 375-391.

3. Edgar Reich, Quasiconformal mappings of the disk with given boundary values, Lecture Notes in Math., vol. 505, Springer-Verlag, Berlin and New York, 1976, pp. 101-135.

4. G. C. Sethares, The extremal property of certain Teichmüller mappings, Comment. Math. Helv. 43 (1968), 98-119.

5. Kurt Strebel, Zur Frage der Eindeutigkeit extremaler quasikonformer Abbildungen des Einheitskreises, Comment. Math. Helv. 36 (1962), 306-323.

6. Marvin Ortel, Extremal quasiconformal mappings with angular dilatation, Indiana Univ. Math. J. 31 (1982), 435-447.

FORSCHUNGSINSTITUT FÜR MATHEMATIK, ETH-ZENTRUM, CH-8092, ZÜRICH, SWITZERLAND

Current address: Department of Mathematics, University of Minnesota, Minneapolis, Minnesota 55455 